\title{
Implicações da Área das Biomédicas na formação do psicólogo para o SUS
}

\author{
Implications of the Biomedical Area in the education of SUS psychologist
}

Neuza Maria de Fátima Guareschi ${ }^{[a]}$, Denise Santos Machry ${ }^{[b]}$, Carolina dos Reis ${ }^{[c]}$, Gisele Dhein ${ }^{[d]}$, Thais Bennemann ${ }^{[e]}$

\begin{abstract}
Resumo

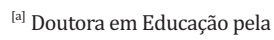
University of Wisconsin-Madison, professora adjunta da Universidade Federal do Rio Grande do Sul (UFRGS), Porto Alegre, RS - Brasil, e-mail: nmguares@gmail.com

${ }^{[b]}$ Psicóloga pela Pontifícia Universidade Católica do Rio Grande do Sul (PUCRS), residente da Saúde da Família e Comunidade, pela Residência Integrada em Saúde do Grupo Hospitalar Conceição (GHC) Porto Alegre - RS, Brasil, e-mail: denise.giz.machry@gmail.com

${ }^{[c]}$ Mestranda do Grupo de Pesquisa Estudos Culturais e Modos de Subjetivação, do Programa de Pós-Graduação em Psicologia Social e

Este artigo tem como objetivo apresentar uma análise sobre o currículo em Psicologia, buscando demonstrar como a formação neste campo de conhecimento se apresenta para atender à demanda colocada pelo Ministério da Saúde de formar profissionais para o SUS. Primeiramente, foi feito um mapeamento das grades curriculares de seis cursos de Psicologia do Estado do Rio Grande do Sul. Apoiando-se em materiais bibliográficos sobre a criação e desenvolvimento dos cursos de Psicologia, identificamos três possíveis eixos de disciplinas que remetem direta ou indiretamente às temáticas da saúde. Neste artigo, apresentamos a análise e discussão somente sobre o eixo que denominamos Área das Biomédicas. Realizamos uma análise das ementas, conteúdos e bibliografias dos programas das disciplinas que propõem o estudo da Área das Biomédicas. A análise desses materiais fundamenta-se na literatura que discute os princípios, diretrizes e concepção de saúde propostos pelo SUS: no princípio da integralidade, nas transformações do conceito de saúde, e na distinção entre saúde pública e saúde coletiva. A partir da análise dos programas das disciplinas, emergiram três enfoques temáticos sobre os quais organizamos a discussão: Sistemas Orgânicos, Genética e Psiquiatria.
\end{abstract} Institucional da Universidade Federal do Rio Grande do Sul (UFRGS), Porto Alegre, RS - Brasil, e-mail carolinadosreis@gmail.com

${ }^{[d]}$ Mestre em Psicologia, área de concentração em Psicologia Social, pela Pontifícia Universidade Católica do Rio Grande do Sul (PUCRS), docente do Centro Universitário Univates, Porto Alegre, RS - Brasil, e-mail: gidhein@gmail.com

${ }^{\left[{ }^{e]}\right.}$ Psicóloga pela Pontifícia Universidade Católica do Rio Grande do Sul (PUCRS), residência em Saúde da Família e Comunidade, pela Residência Integrada em Saúde do Grupo Hospitalar Conceição (GHC), Porto Alegre, RS - Brasil, e-mail: thais. bennemann@gmail.com

Recebido: $26 / 08 / 2010$ Received: 08/26/2010

Palavras-chave: Currículo. Psicologia. Profissional de saúde. SUS. Formação em Psicologia.

\begin{abstract}
This article aims to present an analysis of Psychology curriculum, in an attempt to show how education in this knowledge field has satisfied the demand for SUS professionals required by the Ministry of Health. Firstly, curricula of six Psychology courses in Rio Grande do Sul were mapped. Based on bibliographical materials about the creation and development of Psychology courses, we identified three possible areas of academic subjects that either directly or indirectly led to themes related to health. Secondly, we carried out an analysis of summaries, contents, and bibliographies of subjects that propose the study of those areas. The analysis of these materials was grounded on literature discussing principles, guidelines and conception of health as proposed by SUS: on the principles of integrity, on the trasformations on the concept of being healthy, and the difference between public health and colective health. From the analysis of the programs of subjects emerged three themes upon which we directed our discussion: organic systems, genetics and psychiatry. This article presents an analysis and discussion only on the axis of the area that we have called Biomedical area.
\end{abstract}

Keywords: Curriculum. Psychology. Health Professionals. SUS (Brazilian Public Health System). Graduate education in psychology. 


\section{Introducão}

A formação em Psicologia vem sofrendo alterações nos últimos anos, e é consenso entre os Ministérios da Educação e Ministério da Saúde que a educação dos profissionais está se modificando para atuar nos serviços voltados à atenção à saúde da população brasileira. Dentre essas mudanças, salienta-se a aproximação da Psicologia às necessidades sociais, a inserção dessa área na proposta do Ministério da Saúde de formação de profissionais para o Sistema Único de Saúde (SUS), e a instituição das Diretrizes Curriculares Nacionais (DCNs) da Psicologia, contemplando os objetivos do SUS.

A partir da Constituição de 1988 (Brasil, 1988), com a mudança do paradigma da saúde, sua concepção passa a ser entendida como direito do cidadão e dever do Estado, como uma política integral e plural, e não mais como ausência de doença ou de sintomas, como uma questão coletiva, e não pública, já que o público remete a modelos de programas assistencialistas e a saúde coletiva objetiva a promoção das condições de vida com a participação social. A saúde coletiva vem romper com o modelo biologicista, visando a atenção integral à saúde. Assim, é de fundamental importância a aproximação da Psicologia dos princípios do SUS, de maneira que contemple todas as exigências dessa formação integral em saúde, conforme proposta pela constituição dessas políticas.

Dessa forma, o presente estudo tem como objetivo geral discutir a formação em Psicologia, buscando compreender como os conteúdos das disciplinas do eixo da Área das Biomédicas ${ }^{1}$, presentes nos currículos dos cursos de graduação em Psicologia, vêm viabilizando a formação dos profissionais dessa área para o SUS. Assim, este artigo apresenta, em um primeiro momento, uma discussão teórica sobre saúde pública, saúde coletiva e aproximação da Psicologia ao conceito de saúde.

\section{Concepção de saúde e aproximação com a Psicologia}

Embora os termos "saúde pública" e "saúde coletiva" sejam empregados muitas vezes como sinônimos, saúde pública e saúde coletiva constituem-se como campos não homogêneos. A saúde pública constituiu-se no fim do século XVII, marcando o investimento da medicina nas enfermidades (Birman, 1991), e tem como estratégias básicas a pesquisa e o mapeamento da epidemiologia no espaço urbano, adotando medidas sanitárias para combater as epidemias. A saúde coletiva, em direção oposta à saúde pública, "se constituiu através da crítica sistemática do universalismo naturalista do saber médico" (Birman, 1991, p. 9). Está intimamente associada à entrada das ciências humanas na saúde, que passam a criticar categorias universalizantes da saúde pública, destacando as dimensões simbólicas, éticas e políticas, de forma a relativizar o discurso biológico.

Fundamentado em princípios e diretrizes, o SUS propõe uma transformação na concepção do conceito de saúde; entendido até então pela presença ou ausência de doenças ou sintomas, passa a compreender as questões de saúde como direito do cidadão para promoção das condições de vida e relaciona-se aos modos de ser e estar no mundo, ou seja, saúde como um dispositivo dos modos de subjetivação (Medeiros, Bernardes \& Guareschi, 2005).

Medeiros et al. (2005) enfatizam a importância, para as práticas psicológicas, de entender a saúde como dispositivo na produção de modos de subjetivação a partir da nova concepção de saúde proposta pelo SUS. Essa importância é destacada, uma vez que o conceito de saúde não existiu nas teorias e práticas da Psicologia desde seu início como um campo de saber científico. A Psicologia surgiu no fim do século XIX como uma disciplina que estudava a consciência, visando a explicar como as pessoas se comportavam e porque se comportavam de tal maneira. Sua proposta teórica sustentava-se em pesquisas laboratoriais realizadas com sujeitos não adoecidos. Dessa forma, não se relacionava com as discussões do campo da saúde.

A partir da década de 1970, nota-se uma conversão considerável de psicólogos para o campo da assistência à saúde pública (Dimenstein, 1998). É principalmente com o movimento de desospitalização e de extensão dos serviços de saúde mental à rede básica de saúde que se dá a inserção do psicólogo na saúde pública (Spink, 2003). Assim, a entrada do psicólogo no setor público de saúde acontece ao mesmo tempo em que o modelo médico-assistencial privatista se encontra no auge, mas também

1 Entende-se por Área das Biomédicas o grupo de disciplinas que se propõe ao estudo de conteúdos da Biologia e das áreas médicas, como Anatomia, Fisiologia, Endocrinologia, Neurologia, Genética, Psiquiatria e Farmacologia. 
com os reflexos do esgotamento de investimentos de décadas anteriores. Atualmente, critica-se o modelo asilar, e dá-se ênfase à formação de equipes multiprofissionais (Dimenstein, 1998).

Em 1986, a VIII Conferência Nacional de Saúde (Brasil, 1987), evento que definiu as bases do projeto para a construção do SUS, serviu para incrementar o processo de ampliação da diversidade de profissionais no campo da saúde. Com esse movimento, pela primeira vez a saúde deixa de ser concebida como estado biológico abstrato de normalidade ou de ausência de doença e passa a ser concebida como resultado de um conjunto de condições coletivas de existência, como expressão ativa de um direito de cidadania (Dimenstein, 1998).

Spink (2003), seguindo essa linha, define a Psicologia Social da saúde como comprometida com os direitos sociais a partir de uma ótica coletiva, portanto, fugindo das perspectivas tradicionais ou intraindividuais. Dessa forma, a inserção do profissional de Psicologia nas práticas de saúde coletiva deve-se tornar uma atenção prioritária para a formação nessa área, pois, na história da constituição desse saber enquanto ciência, o conceito de saúde foi sendo integrado nas suas teorias e práticas. Ainda, diante da relevância social do campo da saúde coletiva e das políticas públicas de saúde, a Psicologia como área da saúde - tem o compromisso de repensar sua prática e de direcionar-se para a formação de profissionais para atuarem nesse campo.

\section{Psicologia e formação de profissionais da saúde}

A formação de profissionais na área da saúde, na qual também se encontra a Psicologia, ainda se volta para a abordagem clássica da formação em saúde, em que o ensino é preocupado com a sofisticação dos procedimentos, do conhecimento dos equipamentos auxiliares do diagnóstico, tratamento e cuidado, organizado a partir do referencial técnico-científico acumulado pelos professores em suas respectivas áreas de especialidade (Ceccim \& Feuerwerker, 2004). 0 compromisso em formar profissionais para atuar no SUS tem sido feito por algumas universidades como algo em caráter experimental, como a inclusão de disciplinas da área da saúde, e a admissão de estágios curriculares nesta área. Entretanto já se observa também um movimento nacional com a finalidade de estabelecer normas para a formação e o trabalho dos profissionais da saúde brasileiros, cumprindo a determinação de que a competência para ordenar a formação de recursos humanos na área da saúde é do SUS (Brasil, 1988, art. 200, inciso III).

É parte desse movimento a criação das DCNs dos cursos de graduação, em 1996, em decorrência da Lei de Diretrizes e Bases da Educação Nacional (LDBEN), em oposição ao formato dos currículos mínimos (Brasil, 1996). As DCNs, incluindo o curso de Psicologia a partir da Resolução de 2004, incentivam a maior flexibilização dos desenhos curriculares, a liberdade para organizar as atividades de ensino e a diversidade das formações pela ampla participação dos alunos nas realidades locais de saúde e ativa participação estudantil (Brasil, 2004; Ceccim \& Carvalho, 2005).

Entre os anos de 2001 e 2004, foram aprovadas as DCNs para os cursos de graduação da área da saúde, firmando nacionalmente que a formação desses profissionais deve contemplar o sistema de saúde vigente no país, o trabalho em equipe e a atenção integral à saúde (Brasil, 1990, 2009). A formação em Psicologia define a atenção à saúde como participação na prevenção, promoção, proteção e reabilitação em saúde, segundo referenciais profissionais. A Psicologia definiu a atenção à saúde como seu objetivo específico, além daqueles já considerados essenciais para a formação de um profissional de psicologia (Brasil, 2004).

0 desafio colocado à formação de recursos humanos para o SUS é formar profissionais capazes de promover ações de saúde integral. A integralidade na atenção à saúde, como princípio do SUS, deve ser eixo norteador das necessidades de mudança na formação dos profissionais dessa área de conhecimento e contribuir com a formulação de uma política do SUS para a mudança na graduação das profissões de saúde (Brasil, 1990; Ceccim \& Feuerwerker, 2004), uma vez que predomina o referencial positivista, centrado no modelo orgânico, voltado para a intervenção assistencial em detrimento da prevenção e promoção das condições de vida, intervenções que visam evitar o surgimento de patologias e possibilitar condições de autonomia para os sujeitos. (Campos, 2006; Czeresnia, 2003). A forma biomédica de se relacionar com a saúde e a ausência da discussão das políticas públicas de saúde na formação em Psicologia devem ser colocadas em questão por vários motivos:

Primeiro, era (é) a oportunidade de transformações no perfil de formação em Psicologia, rompendo com

Psicol. Argum. 2012 jul./set., 30(70), 503-513 
modelos individualizantes; segundo, tendo em vista as modificações nas demandas de trabalho que vêm ocorrendo, é importante que o psicólogo se prepare já em sua graduação (vale dizer que o público não se restringe ao estatal); terceiro, refletir criticamente sobre as políticas públicas e o saber-agir da Psicologia atende aos anseios de profissionais que já trabalham com essa questão, além do anseio de usuários dos serviços (Bernardes, 2006, p. 4).

A formação de recursos humanos para atuar no SUS foi um dos temas centrais da Terceira Conferência Nacional de Gestão do Trabalho e da Educação na Saúde (CNGTES), realizada pelo Ministério da Saúde e Conselho Nacional de Saúde em Brasília, em março de 2006. A CNGTES teve como referência que as instituições formadoras devem responsabilizar-se pela produção científica e qualificação de quadros profissionais e, também, tornar-se parceiras do SUS. Devem efetivar a máxima interação entre o mundo do ensino e o mundo do trabalho, consolidando os compromissos públicos e a elevância social da formação, especialmente em saúde, onde existe mandato constitucional para a ação conjunta entre saúde e educação (Brasil, 2006).

\section{Análise de currículos do curso de Psicologia}

A regulamentação da profissão do psicólogo acontece em um momento em que a Psicologia busca um caráter técnico, e tem como principal campo de trabalho o atendimento individual. A partir da década de 1980, com o crescente empobrecimento da população, a Psicologia passa a repensar seu modelo de intervenção e, em consequência, de formação. Esse percurso de alterações nos currículos para adequar-se às demandas sociais se fortalece com o documento chamado "Carta de Serra Negra". Esse documento foi base para a construção das DCNs para os cursos de graduação em Psicologia, no ano de 2004 (Bernardes, 2006).

Alguns cursos da área da Psicologia, para ir ao encontro das ênfases descritas nas DCNs, vêm inserindo nos currículos a formação em saúde como modo de aproximação à proposta do SUS, a partir da modificação de suas bases curriculares. Ainda, em conformidade com o que as DCNs estabelecem como a primeira das competências e habilidades gerais que devem ser trabalhadas pelos cursos de graduação em Psicologia.
Atenção à saúde: os profissionais devem estar aptos a desenvolver ações de prevenção, promoção, proteção e reabilitação da saúde psicológica e psicossocial, tanto em nível individual quanto coletivo, bem como a realizar seus serviços dentro dos mais altos padrões de qualidade e dos princípios da ética/bioética.

Este trabalho propõe-se a mostrar uma análise dos currículos de seis cursos de graduação do Estado do Rio Grande do Sul, sendo duas instituições confessionais, duas federais e duas comunitárias. A escolha dos currículos das seis universidades para esta pesquisa justifica-se por estas terem os cursos de Psicologia há mais de dez anos, o que implica terem formado profissionais no modelo anterior às DCNs dos cursos de Psicologia de 2004. A escolha do currículo desses cursos de Psicologia se deu também por já possuírem egressos em formação nas Residências Multiprofissionais em Saúde (RMS) no Rio Grande do Sul, o que pode demonstrar um movimento em relação à formação de profissionais para o SUS.

Dos cursos de Psicologia selecionados para a realização do estudo, cinco deles propõe dez semestres de formação, e um deles propõe nove semestres. Desse modo, realizamos primeiramente o mapeamento das grades curriculares dos seis cursos de Psicologia, selecionados apenas a partir de materiais retirados dos portais da internet das universidades, e identificamos as disciplinas que, a partir de sua nomenclatura, remetem direta ou indiretamente às temáticas da saúde.

Após esse levantamento, buscamos materiais bibliográficos sobre a história da estruturação e formação dos cursos de Psicologia, buscando entender o modo e o momento em que as disciplinas selecionadas como aquelas que remetem direta ou indiretamente à saúde foram sendo inseridas nos currículos de Psicologia. Esta análise buscou entender as condições para que determinados conhecimentos integrassem a formação em Psicologia, o que nos possibilitou agrupar as disciplinas em três eixos: eixo da Psicologia Social e Comunitária, eixo da Psicopatologia e Avaliação Psicológica e eixo da Área das Biomédicas.

Neste texto, apresentamos somente a análise e discussão das disciplinas que foram agrupadas no eixo que denominamos como Área das Biomédicas - aquelas que remetem, em sua nomenclatura, a estudos das áreas da Anatomia, Fisiologia, Endocrinologia, Neurologia, 
Genética, Psiquiatria, Farmacologia, entre outras. A análise dos eixos da Psicologia Social e Comunitária e da Psicopatologia e Avaliação Psicológica geraram discussões que são apresentadas em outras publicações ${ }^{2}$. Assim, entendemos que a presença dos conteúdos das disciplinas do eixo da Área das Biomédicas vem sendo sustentada na formação em Psicologia a partir de seis fatores (Camargo, 2005, 2007):

- A saúde pública no Brasil - os serviços, os programas e o conhecimento - constituiu-se a partir da medicina moderna, fundamentada pelo discurso das ciências positivistas, oriunda do modelo de medicina social da Inglaterra do fim do século XIX;

- O campo científico da medicina passou a ser detentor de todo o saber sobre saúde, sendo legitimado a afirmar o que é saúde e o que é doença, por meio de pesquisas epidemiológicas, ignorando aspectos históricos e culturais sobre saúde;

- A organização do saber médico estruturou-se a partir dos sistemas teoricamente divididos e subdividiu-se em disciplinas especializadas;

- Como esses saberes se cruzam de forma imperfeita e não correspondem, na sua totalidade, às subdivisões estabelecidas, outros saberes sobre o funcionamento do corpo humano não selecionados anteriormente foram demandados para explicar, agrupar e legitimar o que foge ao cientificismo ${ }^{3}$ da medicina;

- A Psicologia foi chamada para o trabalho na saúde a partir dessa demanda da medicina: como um saber de uma disciplina que se sobrepõe a outras, resultando em mais verdades sobre o sistema científico do conhecimento da medicina, e não como um campo de saber que se coloca para interlocuções ou produções de outras práticas em saúde;

- A Psicologia validou-se como campo científico na ciência da modernidade, legitimando-se também por meio da apropriação de um saber da biologia, no desenvolvimento de práticas em saúde junto à medicina e, posteriormente, na saúde pública.
Para a realização da análise, buscamos identificar como os programas das disciplinas do eixo da Área das Biomédicas dos currículos dos cursos de Psicologia selecionados se articulam com a proposta de saúde do SUS. Os materiais utilizados para análise nessa etapa da pesquisa foram disponibilizados pelas universidades mediante apresentação do projeto, e aprovação pelo Comitê Científico de Pesquisa da Faculdade de Psicologia da Pontifícia Universidade Católica do Rio Grande do Sul (PUCRS), de acordo com Resolução n. 196/96 do Conselho Nacional de Saúde - Ministério da Saúde.

\section{A Área das Biomédicas: 0 corpo e seu funcionamento}

Algumas disciplinas apresentam os sistemas orgânicos do corpo humano e têm seus programas organizados a partir do estudo de conteúdos que correspondem às principais partes do corpo. Dentre essas, o conteúdo das demais disciplinas remete ao estudo de um sistema orgânico específico: Neurologia e Endocrinologia. Outras disciplinas apresentam conteúdos da genética humana, voltando-se para o estudo de como são transmitidas as características biológicas de geração para geração. Por último, algumas disciplinas tratam da Psiquiatria e da Farmacologia e têm seus conteúdos voltados ao estudo de doenças mentais e ao seu tratamento. Dessa forma, a análise e discussão das disciplinas da Área das Biomédicas foram organizadas em três enfoques temáticos, intitulados: Sistemas Orgânicos, Genética e Psiquiatria.

\section{Sistemas orgânicos}

Neste enfoque temático, discutimos o conteúdo dos programas que se propõem ao estudo do organismo humano no ensino da graduação em Psicologia. Os sistemas orgânicos fazem parte da morfoarquitetura anatômica do organismo humano e são responsáveis pelos seus processos funcionais. Dentre esses sistemas orgânicos, estão: o articular, o circulatório, o digestório, o endócrino, o esquelético, o motor, o

\footnotetext{
2 Em artigos submetidos para publicação em revistas científicas e em capítulos do livro Guareschi, N., Scisleski, A., Reis, C., Dhein, G., \& Azambuja, M. (Org.). (2010). Psicologia, formação, política e produção em saúde. Porto Alegre: EDIPUCRS.

${ }^{3}$ Utilizamos aqui o termo cientificismo para denotar uma determinada perspectiva da ciência que considera os conhecimentos científicos definitivos e nega a importância de temas que não são abordados pela alçada científica.
}

Psicol. Argum. 2012 jul./set., 30(70), 503-513 
muscular, o nervoso, o reprodutor, o renal, o respiratório, o sensorial, o tegumentar e o urinário.

Essas disciplinas focam a aprendizagem a partir de quatro pontos: a Anatomia Humana, que estuda a forma e a posição exatas, o tamanho e a relação entre as várias estruturas do corpo humano; a Fisiologia Humana, que estuda o funcionamento, as funções mecânicas, físicas e bioquímicas do organismo humano; a Endocrinologia, que estuda o funcionamento dos hormônios no organismo humano; e a Neurologia, que estuda o sistema nervoso, suas relações e transtornos. Porém, diferentemente do foco de ensino da Anatomia Humana e da Fisiologia Humana, que propõem o aprendizado do organismo de forma geral, as disciplinas de Endocrinologia e Neurologia propõem-se ao ensino exclusivo do seu sistema orgânico correspondente. Estes sistemas orgânicos são os únicos eleitos para serem estudados de forma aprofundada na formação do psicólogo.

Cada um dos pontos de estudo dos sistemas orgânicos - Anatomia Humana, Fisiologia Humana, Endocrinologia e Neurologia - tem, em geral, uma única disciplina correspondente nos currículos pesquisados. Em alguns cursos, um desses pontos não é estudado, como, por exemplo, a Anatomia Humana, a Fisiologia Humana e a Endocrinologia. Já o estudo da Neurologia é proposto por todos os cursos pesquisados, em uma ou duas disciplinas do currículo.

As disciplinas de Endocrinologia e Neurologia relacionam-se diretamente aos sistemas orgânicos correspondentes e recebem, inclusive, o nome a partir de sua especialidade relativa na área médica. Ou seja, essa questão da especialidade colocada na aprendizagem remete ao conhecimento da sociedade moderna, pois a ordem do saber surge como a forma de oferecer veracidade. Bauman (1999, p. 12) refere que "podemos pensar a modernidade como um tempo em que se reflete a ordem". Dessa forma, classificar e delimitar são maneiras de organizar o conhecimento, legitimando-o. É a partir da necessidade de clarificar e dividir os saberes que surgem as especialidades nessa área. No caso das disciplinas, na divisão do corpo humano em organismos e na constituição das áreas do conhecimento que se ocupam de cada uma das especialidades.

As disciplinas que trabalham conteúdos da Anatomia Humana e da Fisiologia Humana abordam os sistemas orgânicos de forma geral em sua proposta de ensino. Por exemplo, as disciplinas de Fisiologia Humana dos primeiros, segundos e terceiros semestres propõem estudar determinados conteúdos dos sistemas do organismo, como o nervoso, o cardiovascular (circulatório), o respiratório, o digestório e o renal. Outros itens das disciplinas de Fisiologia Humana fazem referência a conteúdos mais específicos, como: sinapses e neurotransmisso$\mathrm{res}^{4}$ ao sistema nervoso, junção neuromuscular aos sistemas nervoso e muscular, controle reflexo ao sistema circulatório ou cardiovascular.

Esse exemplo mostra que os sistemas orgânicos são descritos nos programas de forma que não necessitam ser articulados com os outros sistemas do organismo. Apenas em algumas exceções os conteúdos das disciplinas visam a trabalhar a articulação de apenas dois dos sistemas, como é o caso do exemplo citado anteriormente, referente ao item junção neuromuscular, que faz referência aos sistemas nervoso e muscular. Essa não articulação do estudo entre os diferentes sistemas orgânicos evidencia um processo de aprendizagem em que os conteúdos são ensinados de forma fragmentada e dissociada. Esse processo também restringe a possibilidade de discussão sobre os conteúdos ensinados como passíveis de ser transversalisados no entendimento de um sistema orgânico para outro, ou na veiculação desses conteúdos em mais de um sistema, ou seja, cada conteúdo restringe-se à compreensão de um único sistema. Assim, a proposta de um olhar integrado para o organismo humano, conectado entre os seus diversos sistemas, fica prejudicada.

Já disciplinas de Endocrinologia e Neurologia são ministradas nos semestres seguintes ou simultâneos aos semestres de Anatomia Humana e Fisiologia Humana, variando entre os primeiros e terceiros semestres, dependendo de cada curso. Porém, essas disciplinas que elencamos como do enfoque temático dos Sistemas Orgânicos não se relacionam com as outras disciplinas dos enfoques temáticos da Genética ou da Psiquiatria, pois seus conteúdos não fazem nenhuma referência e essas temáticas. Estes são indícios de que o conteúdo dos programas das disciplinas da Área das Biomédicas se coloca de forma fragmentada, não contribuindo para uma formação que possa discutir o funcionamento do corpo humano de forma integrada.

${ }^{4}$ As frases e nomenclaturas referidas em itálico neste capítulo referem-se aos nomes de disciplinas e a seus conteúdos, retirados, de forma literal, dos programas das disciplinas enviados pelas universidades. 
Outro exemplo disso são as disciplinas que fazem referência ao ensino do sistema orgânico nervoso, que remetem seu conteúdo ao estudo deste único sistema, não apresentando outros conteúdos trabalhados pelas demais disciplinas da Área das Biomédicas. Nos programas das disciplinas de Neurologia dos primeiros, segundos e terceiros semestres, são ensinados conteúdos apenas de caráter neurológico, ou seja, sua organização, funcionamento, desenvolvimento, técnicas de exame, intervenção, síndromes e reabilitação.

Dessa forma, deparamo-nos com dois pontos que vêm de encontro à concepção de aprendizagem mais integral que possa articular diferentes conteúdos: primeiramente, o olhar lançado sobre uma única dimensão do corpo humano, que é a mente; em segundo lugar, além de olhar somente para uma dimensão, o entendimento sobre ela parte de uma única perspectiva teórica: a da Neurologia.

Quanto ao sistema endócrino, somente dois currículos dos pesquisados apresentam disciplinas de Endocrinologia na formação do psicólogo, sendo que os conteúdos dessas disciplinas fazem relações entre esse sistema e o psiquismo humano. Podemos perceber a relação proposta - da Endocrinologia com a Psicologia - no conteúdo das disciplinas de Endocrinologia dos segundos semestres. Por exemplo, na ementa de uma das disciplinas, é feita a referência aos sintomas psicológicos de doenças endocrinológicas. Embora disciplinas de Endocrinologia apresentem uma sugestão de relação entre os conteúdos dos dois tópicos de estudo - o sistema endócrino e o psiquismo humano -, é indicada uma proposta de funcionamento distinta para cada um desses sistemas, apontando que o problema endócrino possui uma causa e tratamento específico. Da mesma forma acontece com o psiquismo, ou seja, não é colocada uma possível relação entre esses dois tópicos. É indicado, no que se refere ao funcionamento do organismo do indivíduo, que os sintomas podem ser manifestados por ambas as vias endócrina e psíquica -, porém, a causa é originada somente por uma delas.

\section{Genética}

Neste enfoque temático, apresentamos o conteúdo dos programas que realizam o estudo da natureza do material hereditário que é transmitido pelos genes a partir das gerações. As disciplinas que abordam conteúdos da Genética Humana são ministradas entre os primeiros e segundos semestres do curso de Psicologia dos currículos pesquisados.

Nas disciplinas de Genética Humana, há, por exemplo, conteúdos que dizem do comportamento normal do ser humano e conteúdos referentes ao comportamento anormal. Esses conteúdos não propõem a explicação do que entendem por comportamento normal e anormal. Porém, no item que se refere ao comportamento anormal, vimos a descrição dos estudos sobre transtornos e patologias. Dessa maneira, não se apresentam nos currículos estudados conteúdos que possam indicar uma articulação do ensinamento de comportamento normal e anormal; os dois são vistos de forma separada.

Alguns conteúdos que citam o comportamento normal e anormal, como, por exemplo, principais síndromes que afetam o desenvolvimento normal e o comportamento humano, reforçam a ideia de que, quanto melhor se estabelecerem os critérios para a identificação do que seria um comportamento esperado, mais se reforça a necessidade do ensino desses conteúdos para que indiquem a aprendizagem sobre o que é normal ou anormal. Desse modo, da maneira como estão colocados os conteúdos para a aprendizagem na formação do psicólogo, fica evidenciada uma perspectiva naturalizada sobre aquilo que seria o desenvolvimento e os comportamentos normal e anormal. Esses conteúdos servem como balizadores para o entendimento de comportamentos que não podem ser colocados na categoria do normal e que podem passar a ser entendidos como desviantes, doentios, patológicos, característicos de transtornos, distúrbios ou síndromes.

O conceito de saúde proposto pelo SUS é embasado no contraponto entre saúde pública e saúde coletiva, pois a primeira coloca a saúde como ausência de doença, e a segunda sugere "condições de acesso às possibilidades de vida” (Bernardes, 2004). Assim, a saúde é uma questão plural de direito dos cidadãos, e não mais um domínio das práticas médicas, pois ultrapassa os limites do biológico, passando por atravessamentos sociais. Nesse sentido, é interessante assinalar que alguns conteúdos de disciplinas, ainda do enfoque temático da Genética Humana, ao proporem o ensino de conteúdos na formação em Psicologia, apontam para um caráter de aprendizagem do psicólogo para o possível uso desse conhecimento no exercício de suas funções. 0 modo como é colocada a aprendizagem desses conteúdos está voltada para

Psicol. Argum. 2012 jul./set., 30(70), 503-513 
a aplicação desses conhecimentos na prática. Estas são evidências de um ensino tecnicista, que pode não abrir possibilidades para a flexibilização do conhecimento, necessária para o entendimento da concepção de ser humano integral proposta pelo SUS.

Dessa forma, fica evidenciada, nos conteúdos das disciplinas de Genética Humana, uma aprendizagem do aluno de Psicologia com um caráter tanto naturalizado quanto vinculado a uma perspectiva utilitarista do conhecimento. Os conteúdos são propostos como aqueles necessários para que o psicólogo possa desenvolver as suas práticas e também como aqueles saberes que vão determinar sempre, e do mesmo modo, como lidar com algumas características do desenvolvimento do organismo e da constituição psíquica do indivíduo. Na ementa de uma das disciplinas de Genética Humana, encontramos a proposta da atuação do psicólogo no serviço de aconselhamento genético, não no sentido de abrir para outras possibilidades de inserção do profissional, mas pressupondo uma atividade natural no seu espaço de atuação. Isso pode implicar em uma visão de adequação de um núcleo de competência da Psicologia a diferentes campos de atuação, como se o conhecimento fosse generalizável para qualquer área, sem problematizar a forma como este conteúdo está sendo ensinado e as implicações da inserção da Psicologia nesta área.

\section{Psiquiatria}

No enfoque temático da Psiquiatria, expomos o conteúdo das disciplinas que se propõem ao estudo da prevenção, do atendimento, do diagnóstico, do tratamento e da reabilitação das doenças mentais e também o conteúdo das disciplinas de Farmacologia, que estudam como essas doenças reagem às substâncias químicas usadas para tratá-las. A especialidade da Psiquiatria visa ao bem-estar do indivíduo e ao alívio do sofrimento psíquico, podendo utilizar-se, para esses fins, do tratamento psicoterápico e/ou de medicamentos baseados nos conhecimentos da Farmacologia. É nos fundamentos da Psiquiatria e da Farmacologia como o estudo daquilo que pode vir a aliviar o sofrimento que se encontra a justificativa do ensino desses conteúdos nos currículos de Psicologia.
Somente três dos currículos analisados possuem disciplinas que se propõem o estudo da Psiquiatria. Entretanto os conteúdos dessa especialidade são estudados em mais de uma disciplina em alguns desses currículos. Em relação à Farmacologia, todos os cursos de Psicologia pesquisados possuem uma única disciplina que se remete a esse estudo. As disciplinas de Psiquiatria e de Farmacologia estão colocadas entre os terceiros e sextos semestres nos currículos, ou seja, são propostas em um momento posterior ao das disciplinas relacionadas às temáticas dos Sistemas Orgânicos e da Genética.

Os conteúdos das disciplinas de Psiquiatria ministradas entre o quinto e sexto semestres tratam do ensino do diagnóstico diferencial ${ }^{5}$ como a possibilidade do aprendizado da causa da patologia, não evidenciando uma intencionalidade em articular ou integrar a discussão sobre a patologia. Como exemplo, o conteúdo que propõe realizar diagnóstico diferencial da patologia orgânica da Psiquiatria e da Clínica Médica Geral, indica a necessidade de diagnóstico diferencial para definir a causa e também a especialidade médica que deverá realizar seu tratamento. Esse ensino vem para reforçar a categorização e classificação do que é respectivo a uma doença com certa causa para outra doença de outra causa, ou para a mesma doença com causas diferentes.

Como as disciplinas do enfoque temático da Genética Humana, as disciplinas do enfoque da Psiquiatria também apontam a aquisição de certos conteúdos teóricos e práticos por parte do aluno, com o intuito de capacitar o profissional para determinadas práticas. Exemplo disso são os conteúdos das disciplinas de Psiquiatria, que são elencados de acordo com cada uma das técnicas entendidas como fundamentais para a realização de um diagnóstico por parte do profissional da Psicologia, como exames, entrevistas e elaboração de hipóteses.

Da mesma forma, na ementa de disciplinas que trabalham conteúdos da Farmacologia, entre os terceiros e sextos semestres, é sugerido o estudo do uso da farmacologia na prática clínica. A proposta de ensino nessas disciplinas é de aquisição dos conteúdos programados, em vista de deter informações suficientes para o entendimento, o diálogo e a intervenção com profissionais da saúde da área

\footnotetext{
5 entendimento de diagnóstico diferencial é realizado a partir do campo da Psicologia, que sugere uma busca pela veracidade na validação de hipóteses com base na sintomatologia, visando à eliminação de suspeitas no fechamento do diagnóstico, de acordo com o American Psychiatric Association, 2002.
} 
médica psiquiátrica. Também nas disciplinas de Farmacologia, percebemos, ainda, que os conteúdos são organizados a partir dos grupos de psicofármacos, relacionados diretamente ao seu grupo de patologia correspondente: Benzodiazepínicos (transtornos de ansiedades), Antipsicóticos (síndromes psicóticas), Antidepressivos (síndromes depressivas), Estabilizadores de humor (síndromes afetivas).

\section{Articulação com a proposta do SUS}

A aproximação da Psicologia com a saúde é um movimento que necessita de estranhamento dentro do campo da construção do conhecimento e das práticas psicológicas em saúde. Uma vez que a Psicologia lida com a diversidade, com a multiplicidade das histórias de vida e dos modos de singularização, necessita capturar os diferentes marcadores culturais que circundam o campo social e não colaborar na fragmentação da vida coletiva.

A formação em Psicologia, conforme as DCNs, deve ter seus currículos direcionados também à formação para o SUS. Por isso, o olhar que lançamos aos currículos nesta pesquisa foi o de analisar como estes se apresentam para dar conta da formação do profissional da Psicologia a partir dos princípios do SUS. Neste texto, a partir do material levantado, a análise foi realizada buscando-se identificar como os conteúdos da Área das Biomédicas dos currículos dos cursos de Psicologia se articulam a partir de três principais indicadores: o princípio da integralidade, as transformações propostas para o conceito de saúde, as diferenciações teóricas e políticas entre saúde pública e saúde coletiva.

0 primeiro ponto a ser levantado diz respeito à integralidade. É um conceito bastante discutido na área da saúde coletiva, pois entende que o sujeito deve ser visto de forma integral, fazendo o profissional de saúde olhá-lo não mais somente com os olhos de sua especialidade, mas dialogando com ele e percebendo-o como um todo De acordo com esse princípio, os sujeitos devem ser compreendidos como um todo biopsicossocial. Estabelece-se, assim, a necessidade de um trabalho em equipes multidisciplinares, uma vez que as diversas áreas do conhecimento terão que dialogar para se chegar ao entendimento da atenção à saúde que o SUS propõe. Nesse sentido, uma Psicologia fundamentada no estabelecimento de padrões de comportamento descritivos, que regulamentam o que é o normal e o anormal, assume uma postura disciplinadora de condutas, não focando o olhar para a diferença sob a qual pode compreender as condições de produção em saúde a partir da história singular dos indivíduos.

Nessa esfera, apontamos que os conteúdos programáticos das disciplinas, muitas vezes, não conseguem ter a visão integral dentro do seu próprio ponto de discussão. Algumas disciplinas acabam por não dialogar com outras da mesma área, acusando o quanto as especialidades estão cada vez mais correntes nos campos de conhecimento. Os saberes estão cada vez mais específicos, e, dessa forma, pode-se pensar que passa a ser paradoxal propor um olhar integral ao sujeito. 0 que se apresenta na proposta de aprendizagem é uma integralidade que se fragmenta, pois o saber/fazer do profissional da saúde, neste caso o profissional da Psicologia, é organizado de forma mais específica e/ou limitada.

No que diz respeito ao caráter das especialidades, percebemos que há uma necessidade de demarcar as fronteiras com outros saberes/disciplinas, para deixar claros os limites de cada um/uma. No entanto a proposta do SUS é de um trabalho interdisciplinar, em que a interlocução entre as áreas de conhecimento se torna cada vez mais relevante. Pensando a Psicologia enquanto disciplina da área da saúde e a necessidade para que os currículos se direcionem para a formação desse profissional para o SUS, é constatada ainda uma grande carga horária de disciplinas da Área das Biomédicas, em relação a outras disciplinas que possam dar conta de conteúdos que sejam tratados de forma mais plural, como proposto pelo SUS.

Os conteúdos programáticos ainda se voltam a um entendimento de sujeito "psíquico" deslocado dos contextos históricos e sociais. Assim, levantamos a questão sobre a discussão que essas disciplinas se propõem a realizar. A integralidade, como princípio que norteia as ações em saúde, representa muito mais do que a soma de olhares especializados e efetiva-se somente por meio da composição de um trabalho em equipes multidisciplinares. A construção de uma proposta como essa exige a desconstrução das barreiras das especificidades das áreas do conhecimento e o rompimento das fronteiras identitárias dos seus objetos de estudo.

A proposta do SUS, em contraponto à formação que a Psicologia vinha realizando até a implementação das novas DCNs, é a de contemplar em seu quadro profissional, além da Psicologia, outras áreas

Psicol. Argum. 2012 jul./set., 30(70), 503-513 
das Ciências Humanas. Assim, entramos no segundo ponto, que diz respeito à naturalização da saúde e a concepção de saúde proposta pelo SUS, pautada não mais na simples ausência de doença, mas na busca do profissional de saúde pela promoção das condições de vida dos sujeitos, lançando um olhar integral que abarque os diversos contextos sociais e culturais em que os indivíduos estão inseridos, o que caracteriza a saúde coletiva. Portanto, o que se evidencia nesta pesquisa é uma incompatibilidade da perspectiva da saúde coletiva com o caráter de uma Psicologia mais voltada para o diagnóstico preciso dos transtornos psiquiátricos, reduzindo as possibilidade de encontro de outras formas de produção de saúde.

Neste ponto, entendemos ser relevante apontar a contradição encontrada nos currículos: sendo a proposta dos currículos uma formação para o SUS, o qual trabalha com o conceito de saúde ampliado, em que o foco é o investimento na vida do sujeito e não mais na doença, encontramos um número expressivo de conteúdos e disciplinas que ainda priorizam o enfoque na patologização e na doença. A busca por uma causa no biológico está também fortemente presente nos conteúdos das disciplinas da Área das Biomédicas. Há, ainda, uma formação voltada à normalização do anormal, à cura da doença e à correção do desvio. 0 olhar está para o que foge à norma e não para a potência da vida.

Chegamos, assim, ao terceiro ponto. Na medida em que a saúde coletiva passa a integrar as DCNs, ela surge como derivada da saúde pública. A. Bernardes (2006, p. 125) aponta que a saúde coletiva é aquela que propicia o encontro da Psicologia com a política, "embora não em termos de uma modificação da racionalidade do público, da saúde e da população, e sim como uma forma de resistência, de práticas reflexivas, que a todo o momento convocam novas formas de o poder se exercer".

Neste aspecto também se evidencia uma naturalização das teorias, sendo estas ensinadas como legítimas e "verdadeiras". A perspectiva da problematização do conhecimento é pouco apontada pelos conteúdos das disciplinas. A discussão da construção do saber, de como este se tornou legítimo e da forma como a ciência se produz e se sustenta também não tem ênfase nos programas dos currículos. Uma vez que há uma mudança paradigmática do currículo - que agora também deve se voltar à formação para o SUS -, entendemos que seria relevante um espaço para discutir as transformações históricas da construção do saber e produção da ciência, durante a formação.

Dessa forma, além da dificuldade de ultrapassar as fronteiras disciplinares do conhecimento para a composição das equipes de saúde, existe uma questão que é central no fazer em Psicologia junto ao SUS: a necessidade de um posicionamento ético/político que se impõe na relação com seu objeto de estudo, isto é, com os sujeitos: o de compreendê-los de forma integral, situados em diferentes contextos históricos e sociais.

Nascimento, Manzini e Bocco (2006, p. 16), ao trazerem uma crítica às práticas $p s i$, remetem à história da Psicologia a gênese do pensamento dicotomizado e objetivo. A crença em verdades imutáveis e objetivas diz dessa origem. Dessa maneira, Psicologia e política têm se construído como territórios separados e em oposição. A proposta das novas diretrizes curriculares nos dá condições de existência de outra Psicologia, onde teremos política e Psicologia não mais distantes, e sim propiciando práticas implicadas que atuem na "potência política da vida". Essa perspectiva coloca em xeque as concepções de distanciamento e neutralidade que visam à objetividade e vai ao encontro de outras verdades que precisam ser assumidas pela Psicologia na sua construção como ciência e como área de formação do profissional para o SUS.

\section{Referências}

American Psychiatric Association. (2002). DSM-IV-TR ${ }^{\mathrm{TM}}$ : Manual Diagnóstico e Estatístico de Transtornos Mentais. Porto Alegre: Artmed.

Bauman, Z. (1999). A busca da ordem. In Z. Bauman. Modernidade e ambivalência (pp. 9-26). Rio de Janeiro: J. Zahar.

Bernardes, A. G. (2006). Políticas da existência no campo da saúde: 0 público como dispositivo. Tese de Doutorado. Programa de Pós-Graduação em Psicologia da Pontifícia Universidade Católica do Rio Grande do Sul, Porto Alegre.

Bernardes, J. de S. (2004). 0 debate atual sobre a formação em Psicologia no Brasil: permanências, rupturas e cooptações nas políticas educacionais. Tese de Doutorado, Programa de Estudos Pós-Graduados em Psicologia Social da Pontifícia Universidade Católica de São Paulo, São Paulo. 
Bernardes, J. de S. (2006). Políticas públicas e formação em Psicologia. EntreLinhas, 33(7), 4.

Birman, J. (1991). A Physis da Saúde Coletiva. Physis: Revista de Saúde Coletiva, 1(1), 7-11.

Brasil. Constituição (1988). Constituição: República Federativa do Brasil. Brasília, DF: Senado Federal, 1988.

Brasil. (1990). Lei n. 8.080, de 19 de setembro de 1990. Dispõe sobre as condições para promoção, proteção e recuperação da saúde, a organização e o funcionamento dos serviços correspondentes e dá outras providências. Diário Oficial [da] República Federativa do Brasil, Poder Legislativo, Brasília, DF, 19 set. 1990. Recuperado em 18 fev. 2011, em http://www. planalto.gov.br/ccivil_03/Leis/L8080.htm

Brasil. (1996). Lei n. 9.394, de 20 de dezembro de 1996. Estabelece as diretrizes e bases da educação nacional. Diário Oficial [da] República Federativa do Brasil, Poder Legislativo, Brasília, DF, 20 dez. 1996. Recuperado em 18 fev. 2011, em http://www.planalto.gov.br/ccivil_03/Leis/L9394.htm

Brasil. Ministério da Educação. Conselho Nacional de Educação. (2009). Resoluções do CNE. Recuperado em 18 fev. 2011, em http://portal.mec.gov.br/index. php?option $=$ com_content $\&$ view $=$ article $\& i d=12816$ \&Itemid $=866$

Brasil. Ministério da Educação. Conselho Nacional de Educação. Câmara de Educação Superior. (2004). Resolução n. 8, de 7 de maio de 2004. Institui as Diretrizes Curriculares Nacionais para os cursos de graduação em Psicologia. Recuperado em 18 fev. 2011, em http://portal.mec.gov.br/cne/arquivos/ pdf/rces08_04.pdf

Brasil. Ministério da Saúde. (1987). Anais da 8ª Conferência Nacional de Saúde. Brasília: Centro de Documentação do Ministério da Saúde. Recuperado em 18 fev. 2011, em http://portal.saude.gov.br/portal/ arquivos/pdf/8_CNS_Anais.pdf

Brasil. Ministério da Saúde. (2006). Consolidado dos relatórios das conferências estaduais de gestão do trabalho e da educação na saúde. In Anais do $3^{\mathbf{a}}$ Conferência Nacional de Gestão do Trabalho e da Educação na Saúde. Brasília: Conselho Nacional de Saúde.
Camargo, K. R. Jr. (2005). A biomedicina. Physis: Revista de Saúde Coletiva, 15(Suplemento), 177-201.

Camargo, K. R. Jr. (2007). A razão inconstante: Ciência, saber e legitimação social. In Associação Brasileira de Psicologia Social (Org.). XIV Encontro Nacional da Abrapso (pp. 17-34). Porto Alegre: Evangraf.

Campos, G. W. de S. (2006). Políticas de formação de pessoal para o SUS: Reflexões fragmentadas. Cadernos RH Saúde, 1(3), 55-59.

Ceccim, R. B., \& Feuerwerker, L. C. M. (2004). Mudança na graduação das profissões de saúde sob o eixo da integralidade. Cadernos de Saúde Pública, 5(20) 1400-1410.

Ceccim, R. B., \& Carvalho, Y. M. de. (2005). Ensino da saúde como projeto da integralidade: A educação dos profissionais de saúde no SUS. In R. Pinheiro, R. B. Ceccim \& R. A. de Mattos (Org.). Ensinar Saúde: A integralidade e o SUS nos cursos de graduação na área da saúde (pp. 69-92). Rio de Janeiro: IMS/ UERJ; CEPESQ; ABRASCO.

Czeresnia, D. (2003). 0 conceito de saúde e a diferença entre prevenção e promoção. In D. Czeresnia \& C. M. Freitas (Org.). Promoção da saúde: Conceitos, reflexões, tendências (pp. 39-53). Rio de Janeiro: FIOCRUZ.

Dimenstein, M. D. B. (1998). 0 psicólogo nas unidades básicas de saúde: Desafios para a formação e atuação profissional. Estudos de Psicologia, 1(3), 53-81.

Guareschi, N., Scisleski, A., Reis, C., Dhein, G., \& Azambuja, M. (Org.). (2010). Psicologia, formação, política e produção em saúde. Porto Alegre: EDIPUCRS.

Medeiros, P. F. de, Bernardes, A. G., \& Guareschi, N. M. F. (2005). 0 conceito de saúde e suas implicações nas práticas psicológicas. Psicologia Teoria e Pesquisa, 3(21) 263-269.

Nascimento, M. L., Manzani, J. M., \& Bocco, F. (2006). Reinventando as práticas Psi. Psicologia e Sociedade 1(18), 15-20.

Spink, M. J. (2003). Psicologia social e saúde: Práticas, saberes e sentidos. Petrópolis: Vozes. 